MATHEMATICS OF COMPUTATION

Volume 66, Number 217, January 1997, Pages 125-138

S 0025-5718(97)00799-0

\title{
PRECONDITIONING THE POINCARÉ-STEKLOV OPERATOR BY USING GREEN'S FUNCTION
}

\author{
JINCHAO XU AND SHENG ZHANG
}

\begin{abstract}
This paper is concerned with the Poincaré-Steklov operator that is widely used in domain decomposition methods. It is proved that the inverse of the Poincaré-Steklov operator can be expressed explicitly by an integral operator with a kernel being the Green's function restricted to the interface. As an application, for the discrete Poincaré-Steklov operator with respect to either a line (edge) or a star-shaped web associated with a single vertex point, a preconditioner can be constructed by first imbedding the line as the diameter of a disk, or the web as a union of radii of a disk, and then using the Green's function on the disk. The proposed technique can be effectively used in conjunction with various existing domain decomposition techniques, especially with the methods based on vertex spaces (from multi-subdomain decomposition). Some numerical results are reported.
\end{abstract}

\section{INTRODUCTION}

In the study of nonoverlapping domain decomposition methods, the so-called Poincaré-Steklov operator plays an important role. How to effectively precondition this operator is significant, both from theoretical and practical points of view. There have been basically two known approaches to precondition such an operator: one is by FFT (Fast Fourier Transform) (cf. [6, 12, 2, 1, 3, 4]), and the other by multigrid preconditioner (cf. [17, 15, 16, 5, 19]). These approaches, however, cannot always be conveniently applied, for example, (1) in three dimensions, (2) if the grids on the interface are not very uniform or do not have a natural multigrid structure, (3) the interface consists of several lines or planes that intersect at a point. In this paper, a completely different technique will be proposed, which gives an alternative, and sometimes a better, approach to preconditioning the Poincaré-Steklov operator. This technique can subsequently be combined with existing domain decomposition approaches.

The idea is, for a two-dimensional problem for example, to imbed the underlying interface as a diameter or union of radii of a disk, and then use the restriction of the Green's function associated with a disk. Advantages of this approach include: (1) it does not require much structure of the underlying grid; (2) it applies naturally to domain decomposition with cross points; (3) it is easy to implement.

Received by the editor May 10, 1995 and, in revised form, July 31, 1995 and January 26, 1996. 1991 Mathematics Subject Classification. Primary 65N20, 65F10.

Key words and phrases. Domain decomposition, preconditioner, Schur complement, Green's function, multigrid, Poincaré-Steklov operator.

This work was partially supported by National Science Foundation, Chinese Academy of Sciences and China National Natural Science funds.

(C) 1997 American Mathematical Society 
The preconditioning technique in this paper is based on a new observation that the inverse of the Poincaré-Steklov operator can be expressed explicitly by an integral operator with a kernel being the Green's function restricted to the interface. This observation appears to be of independent theoretical significance.

For clarity, the presentation of the paper will be confined to only two-dimensional problems, but the method can also be extended to three-dimensional problems; such an extension, however, is not very straightforward, and it will be discussed in a future work.

For convenience, following [18], the symbols $\lesssim, ~$ and $\equiv$ will be used in this paper. The relations $x_{1} \lesssim y_{1}, x_{2} \gtrsim y_{2}$ and $x_{3} \equiv y_{3}$ mean that $x_{1} \leq C_{1} y_{1}, \quad x_{2} \geq c_{2} y_{2}$ and $c_{3} x_{3} \leq y_{3} \leq C_{3} x_{3}$, respectively, for some constants $C_{1}, c_{2}, c_{3}$ and $C_{3}$ that are independent of mesh parameters.

The rest of the paper is organized as follows. Section 2 gives a description of the Poincaré-Steklov operator and an integral representation of its inverse; Section 3 studies the discrete Poincaré-Steklov operator and the new preconditioner; Section 4 contains some numerical examples and discussions of the application of the new preconditioner together with some well-known nonoverlapping domain decomposition methods.

\section{Poincaré-Steklov OPERATOR}

Consider the following model boundary value problem:

$$
\begin{cases}r l-\Delta u=f & \text { in } \Omega, \\ u=0 & \text { on } \partial \Omega,\end{cases}
$$

where $\Omega \subset R^{2}$ is a bounded domain. Let $H_{0}^{1}(\Omega)$ be the standard Sobolev space of square integrable functions with square integrable gradients and zero Dirichlet boundary condition, with a norm given by

$$
|u|_{1, \Omega}=\|\nabla u\|_{0, \Omega}, \quad u \in H_{0}^{1}(\Omega) .
$$

The variational form of $(1)$ is to seek $u \in H_{0}^{1}(\Omega)$ satisfying

$$
a(u, v)=(f, v) \quad \forall v \in H_{0}^{1}(\Omega),
$$

with

$$
a(u, v)=\int_{\Omega} \nabla u \cdot \nabla v
$$

To discuss the Poincaré-Steklov operator, two different cases will be considered. The first case is a general bounded domain $\Omega$ which is decomposed into two mutually disjoint subdomains $\Omega_{1}$ and $\Omega_{2}$ such that

$$
\bar{\Omega}=\bar{\Omega}_{1} \cup \bar{\Omega}_{2} ;
$$

$\Gamma=\partial \Omega_{1} \cap \partial \Omega_{2}$ is called the interface of this domain decomposition with two subdomains; see Fig. 1 below in $\S 2.2$.

The second case is a bounded domain $\Omega$ which is decomposed into several mutually disjoint subdomains $\Omega_{1}, \Omega_{2}, \ldots, \Omega_{p}$,

$$
\bar{\Omega}=\bigcup_{i=1}^{p} \bar{\Omega}_{i} ;
$$


furthermore, all $\partial \Omega_{i} \backslash \partial \Omega$ consist of only straight lines that meet at one single point (which is known as the cross-point). Again, $\Gamma=\bigcup_{i=1}^{p} \Gamma_{i}$ (with $\Gamma_{i}=\partial \Omega_{i} \backslash \partial \Omega$ ) is the interface of this domain decomposition.

The discussion in this section, unless specified otherwise, will be devoted to both of the aforementioned cases of domains together with their domain decompositions.

In this paper, the space $H_{00}^{1 / 2}(\Gamma)$ will be defined to be the space consisting of the trace of functions in $H_{0}^{1}(\Omega)$, together with a norm given by

$$
\left|u_{\Gamma}\right|_{H_{00}^{1 / 2}(\Gamma)}=\inf _{u \in H_{0}^{1}(\Omega),\left.u\right|_{\Gamma}=u_{\Gamma}}|u|_{H_{0}^{1}(\Omega)} .
$$

It is easy to see that

$$
\left|u_{\Gamma}\right|_{H_{00}^{1 / 2}(\Gamma)}=\left|u^{H}\right|_{H_{0}^{1}(\Omega)},
$$

where $u^{H} \in H_{0}^{1}(\Omega)$ is the harmonic extension of $u_{\Gamma}$ to all the subdomains, namely $u^{H}$ satisfies

$$
\begin{cases}r l-\Delta u^{H}=0 & \text { in } \Omega \backslash \Gamma, \\ u^{H}=u_{\Gamma} & \text { on } \Gamma .\end{cases}
$$

The space $H^{-1 / 2}(\Gamma)$ will be defined to be the dual space of $H_{00}^{1 / 2}(\Gamma)$, and its norm is given by

$$
\|u\|_{-\frac{1}{2}, \Gamma}=\sup _{v_{\Gamma} \in H_{00}^{1 / 2}(\Gamma)} \frac{\left(u_{\Gamma}, v_{\Gamma}\right)_{0, \Gamma}}{\left|v_{\Gamma}\right|_{H_{00}^{1 / 2}(\Gamma)}} .
$$

It is well known that (see Nečas [13])

$$
\left|u^{H}\right|_{H^{1}\left(\Omega_{i}\right)} \equiv\left|u_{\Gamma}\right|_{H_{00}^{1 / 2}\left(\Gamma_{i}\right)},
$$

where

$$
\left|u_{\Gamma}\right|_{H_{00}^{1 / 2}\left(\Gamma_{i}\right)}^{2}=\left|u_{\Gamma}\right|_{1 / 2, \Gamma_{i}}^{2}+\int_{\Gamma_{i}} \frac{\left|u_{\Gamma}(x)\right|^{2}}{\operatorname{dist}\left(x, \partial \Gamma_{i}\right)} d x .
$$

Here, $|\cdot|_{1 / 2, \Gamma_{i}}^{2}$ is the seminorm in $H^{1 / 2}\left(\Gamma_{i}\right)$ defined as

Consequently,

$$
\left|u_{\Gamma}\right|_{1 / 2, \Gamma_{i}}^{2}=\int_{\Gamma_{i}} \int_{\Gamma_{i}} \frac{\left(u_{\Gamma}(x)-u_{\Gamma}(y)^{2}\right)}{|x-y|^{2}} d x d y .
$$

$$
\left|u_{\Gamma}\right|_{H_{00}^{1 / 2}(\Gamma)}^{2} \equiv \sum_{i=1}^{p}\left|u_{\Gamma}\right|_{H_{00}^{1 / 2}\left(\Gamma_{i}\right)}^{2} .
$$

The norm given in (2) is used in the conventional way of defining $H_{00}^{1 / 2}$ (see Lions and Magenes [10]). The equivalence (3) relates the $H_{00}^{1 / 2}$ space for the cross-like domain to the conventional definition (2).

The Poincaré-Steklov operator $S$ is defined as follows:

$$
\int_{\Gamma}\left(S u_{\Gamma}\right) v_{\Gamma}=a\left(u^{H}, v^{H}\right) \quad \forall v_{\Gamma} \in H_{00}^{1 / 2}(\Gamma)
$$

or, by the Green's formula, formally

$$
S u_{\Gamma}=\left.\left(\frac{\partial u^{H}}{\partial n_{1}}+\frac{\partial u^{H}}{\partial n_{2}}\right)\right|_{\Gamma},
$$


where $n_{1}$ and $n_{2}$ denote the inner normals of $\Gamma$ (excluding the cross-point) with respect to the two neighboring subdomains.

Note that, since $u^{H}$ is harmonic, the defining identity (4) holds for more general functions $v$ which are extensions of $v_{\Gamma}$, namely

$$
\left(S u_{\Gamma}, v_{\Gamma}\right)_{0, \Gamma}=a\left(u^{H}, v\right) .
$$

The basic property of the operator $S$ is given in the following theorem.

Theorem 2.1. The Poincaré-Steklov operator $S$ defined by (4) is a well-defined operator such that

$$
S: H_{00}^{1 / 2}(\Gamma) \rightarrow H^{-1 / 2}(\Gamma) .
$$

Furthermore, $S$ is bijective and symmetric positive definite.

Proof. It follows from (4) that

$$
\left(S u_{\Gamma}, v_{\Gamma}\right)_{0, \Gamma} \leq\left|u^{H}\right|_{1, \Omega}\left|v^{H}\right|_{1, \Omega}=\left|u_{\Gamma}\right|_{\frac{1}{2}, \Gamma}\left|v_{\Gamma}\right|_{\frac{1}{2}, \Gamma} .
$$

Thus, $S: H_{00}^{1 / 2}(\Gamma) \rightarrow H^{-1 / 2}(\Gamma)$ is well defined and

$$
\left\|S u_{\Gamma}\right\|_{-1 / 2, \Gamma}=\left|u_{\Gamma}\right|_{H_{00}^{1 / 2}(\Gamma)} \text { and }\left(S u_{\Gamma}, u_{\Gamma}\right)=\left|u_{\Gamma}\right|_{H_{00}^{1 / 2}(\Gamma)}^{2} .
$$

Clearly $S$ is symmetric positive and definite. On the other hand, for any $f_{\Gamma} \in$ $H^{-1 / 2}(\Gamma)$, consider the following variational problem:

$$
a(u, v)=\left(f_{\Gamma}, v_{\Gamma}\right)_{0, \Gamma} \quad \forall v \in H_{0}^{1}(\Omega) .
$$

Obviously, this problem uniquely determines $u \in H_{0}^{1}(\Omega)$. By (4), $u_{\Gamma}$, the trace of $u$ on $\Gamma$, is the preimage of $f_{\Gamma}$ under $S$. Consequently, $S$ is surjective and invertible, and, in fact, $u_{\Gamma}=S^{-1} f_{\Gamma}$.

2.1. An integral representation for the inverse. As shown above, the operator $S$ maps $H_{00}^{1 / 2}(\Gamma)$ to $H^{-1 / 2}(\Gamma)$ bijectively. In this subsection we demonstrate that the inverse of $S$ can be expressed as an integral operator with a kernel being the restriction of the Green's function to the interface.

Theorem 2.2. Let $G(\cdot, \cdot)$ be the Green's function associated with the problem (1). Then the inverse of the Poincaré-Steklov operator $S$ has the following representation:

$$
\left(S^{-1} f_{\Gamma}\right)(x)=\int_{\Gamma} G\left(x, x^{\prime}\right) f_{\Gamma}\left(x^{\prime}\right) d s\left(x^{\prime}\right) \quad \text { for } \quad f_{\Gamma} \in L^{\infty}(\Gamma) .
$$

Proof. Given $f_{\Gamma} \in L^{\infty}(\Gamma)$, let $u_{\Gamma}=S^{-1} f_{\Gamma}$ and $u^{H}$ be the harmonic extension of $u_{\Gamma}$ to $\Omega \backslash \Gamma$. Note that $u^{H} \in C^{\infty}(\Omega \backslash \Gamma)$. Given $x \in \Omega \backslash \Gamma$, substituting for $v$ in (6) the Green's function $G(x, \cdot)$ associated with (1) yields

$$
u^{H}(x)=\int_{\Gamma} G(x, y) f_{\Gamma}(y) d y .
$$

Note that $G(\cdot, \cdot)$ is nonnegative and $G(x, \cdot) \in L^{1}(\Gamma)$ for $x \in \Gamma$. By using the Lebesgue dominated convergence theorem, and letting $x \in \Omega \backslash \Gamma$ approach $\Gamma$ in the above identity, we conclude that

$$
u_{\Gamma}(x)=\int_{\Gamma} G(x, y) f_{\Gamma}(y) d y \quad \forall x \in \Gamma .
$$

The desired representation then follows by definition. 
Remark 1. The expression (8) is valid for more general functions $f$, but the space $L^{\infty}$ is sufficient for applications to finite element subspaces.

2.2. A spectrally equivalent operator. Naturally, one expects that the explicit expression of $S^{-1}$ given by (8) can be used to construct a preconditioner for the (discrete) Poincaré-Steklov operator, but the problem is that the Green's function $G$ is not easily computable, even for rectangular regions. One way to get around this difficulty is to imbed $\Gamma$ into a domain for which the Green's function is simple and easy to compute. The simplest domain in this regard is a disk. For simplicity of exposition, a unit disk will be considered; its associated Green's function has the following expression:

$$
G^{0}\left(z, z^{\prime}\right)=\frac{1}{4 \pi} \log \frac{1+r^{2}\left(r^{\prime}\right)^{2}-2 r r^{\prime} \cos \left(\theta-\theta^{\prime}\right)}{r^{2}+\left(r^{\prime}\right)^{2}-2 r r^{\prime} \cos \left(\theta-\theta^{\prime}\right)} .
$$

Here, $(r, \theta)$ and $\left(r^{\prime}, \theta^{\prime}\right)$ are the polar coordinates of $z$ and $z^{\prime}$, respectively.

For the two-subdomain case, $\Gamma$ may be identified as the interval $[-1,1]$ on the $x$-axis. The restriction of the above Green's function to $\Gamma$ can be written as

$$
G^{0}\left(x, x^{\prime}\right)=\frac{1}{2 \pi} \log \frac{1-x x^{\prime}}{\left|x-x^{\prime}\right|}, \quad x, x^{\prime} \in[-1,1] .
$$

In the multi-subdomain decomposition, the interface can be composed of unitlength straight lines that meet at the origin $(0,0)$ (Fig. 1).

Under such an assumption about the interface, $G^{0}$ can be used to replace the kernel in the inverse integral operator in (8) to obtain another operator $\tilde{S}$ from $H_{00}^{1 / 2}(\Gamma)$ to $H^{-1 / 2}(\Gamma)$ whose inverse can be expressed as

$$
\tilde{S}^{-1} f_{\Gamma}=\int_{\Gamma} G^{0}\left(\cdot, x^{\prime}\right) f_{\Gamma}\left(x^{\prime}\right) d s\left(x^{\prime}\right) .
$$

Theorem 2.3. For any function $u_{\Gamma} \in H_{00}^{1 / 2}(\Gamma)$, we have

$$
\left(\tilde{S} u_{\Gamma}, u_{\Gamma}\right) \equiv\left(S u_{\Gamma}, u_{\Gamma}\right) .
$$

This equivalence may be dependent on the shape of $\Omega$.
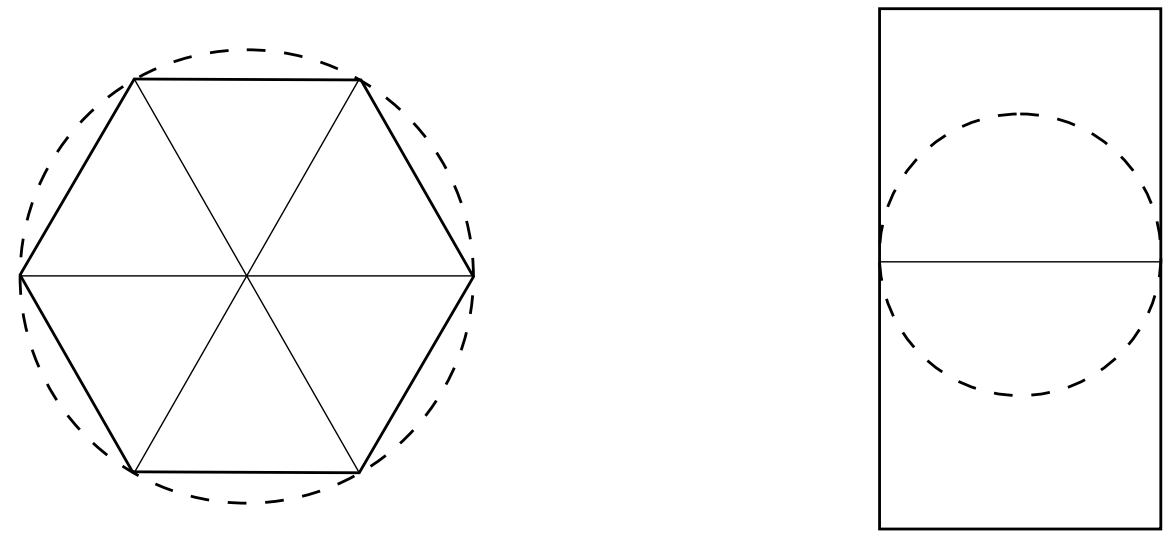

Figure 1. Example of domain decomposition with the interface being a diameter or radii of a disk 
Proof. Let $\Omega^{0}$ be the open unit disk. Under the assumption on $\Gamma, \Omega^{0}$ is decomposed into several mutually disjoint subdomains $\Omega_{1}^{0}, \Omega_{2}^{0}, \ldots, \Omega_{p}^{0}$ by $\Gamma$ as well. By the definition of $\tilde{S}$, it is easy to see that

$$
\left(\tilde{S} u_{\Gamma}, u_{\Gamma}\right)=\left\|u_{\Gamma, 0}^{H}\right\|_{H_{0}^{1}\left(\Omega^{0}\right)}^{2} .
$$

Here, $u_{\Gamma, 0}^{H}$ is the harmonic extension of $u_{\Gamma}$ to $\Omega^{0} \backslash \Gamma$, and we have

$$
\begin{aligned}
\left\|u_{\Gamma, 0}^{H}\right\|_{H_{0}^{1}\left(\Omega^{0}\right)}^{2} & =\sum_{i=1}^{p}\left\|u_{\Gamma, 0}^{H}\right\|_{H^{1}\left(\Omega_{i}^{0}\right)}^{2} \\
& \equiv \sum_{i=1}^{p}\left\|u_{\Gamma}\right\|_{H_{00}^{1 / 2}\left(\Gamma_{i}\right)}^{2} \\
& \equiv \sum_{i=1}^{p}\left\|u_{\Gamma}^{H}\right\|_{H^{1}\left(\Omega_{i}\right)}^{2} \\
& =\left\|u_{\Gamma}\right\|_{H_{00}^{1 / 2}(\Gamma)}^{2} \\
& =\left(S u_{\Gamma}, u_{\Gamma}\right) . \quad \square
\end{aligned}
$$

Thanks to the above theorem, $\tilde{S}^{-1}$ can be used as a preconditioner for the operator $S$.

\section{Discrete Poincaré-Steklov operator in finite Element sPaces}

With the Poincaré-Steklov operator and its inverse studied in the last section for both the two-subdomain decomposition and a special multi-subdomain decomposition, we shall now define their discrete counterparts in finite element spaces and discuss their relationship. These discrete operators in finite element spaces will be applied to precondition the Schur complement matrix in the following subsection.

3.1. Analysis of the discrete Poincaré-Steklov operator. Because of Theorem 2.3, it is sufficient for us to discuss the unit-disk domain $\Omega$. We assume that the interface of a domain decomposition of $\Omega$ is a diameter in the two-subdomain case and/or composed of radii in the multi-subdomain case. Let $T$ be a quasi-uniform finite element triangulation on $\Omega$ which is compatible with $\Gamma$ in such a way that $\Gamma$ can be represented as a union of edges of some elements in $T$. Let $\Omega^{h}$ denote the set of finite element nodal points in $\Omega$ and $\Gamma^{h}=\Omega^{h} \cap \Gamma, V_{0}^{h}(\Omega)$ the finite element function space with $\left\{\phi_{i}, z_{i} \in \Omega^{h}\right\}$ being the set of the usual nodal basis functions, and $V_{\Gamma}^{h}$ the restriction of $V_{0}^{h}(\Omega)$ to $\Gamma$.

Motivated by the variational form (4) of the Poincaré-Steklov operator, we define its discrete counterpart $S_{h}$ in the finite element space $V_{\Gamma}^{h}$ as

$$
\left(S_{h} u_{\Gamma}^{h}, v_{\Gamma}^{h}\right)=a\left(u_{h}^{H}, v_{h}^{H}\right) \quad \forall v_{\Gamma}^{h} \in V_{\Gamma}^{h},
$$

where $u_{h}^{H}$ is the discrete harmonic extension of $u_{\Gamma}^{h}$ to $\Omega$ satisfying

$$
a\left(u_{h}^{H}, \phi_{i}\right)=0 \quad \forall z_{i} \in \Omega^{h} \backslash \Gamma^{h} .
$$

We now define $T_{h}$ in $V_{\Gamma}^{h}$ as the discrete version of $S^{-1}$ as follows:

$$
\left(T_{h} u_{\Gamma}^{h}, v_{\Gamma}^{h}\right)=\left(S^{-1} u_{\Gamma}^{h}, v_{\Gamma}^{h}\right) \quad \forall v_{\Gamma}^{h} \in V_{\Gamma}^{h} .
$$


By Theorem 2.2,

$$
\left(T_{h} u_{\Gamma}^{h}, v_{\Gamma}^{h}\right)=\left(\int_{\Gamma} G^{0}\left(\cdot, x^{\prime}\right) u_{\Gamma}^{h}\left(x^{\prime}\right) d x^{\prime}, v_{\Gamma}^{h}\right) .
$$

Evidently, $S_{h}$ and $T_{h}$ are symmetric positive and definite operators in the finite element space $V_{\Gamma}^{h}$ under the $L^{2}$ inner product. We shall show next that $T_{h}$ is spectrally equivalent to $S_{h}^{-1}$. One technical tool in the proof of such an equivalence is a stability property of the $L^{2}$ projection on the interface.

Lemma 3.1. Let $Q_{h}$ be the $L^{2}$ projection from $L^{2}(\Gamma)$ to $V_{\Gamma}^{h}$. Then

$$
\left\|Q_{h} \phi\right\|_{H_{00}^{1 / 2}(\Gamma)} \lesssim\|\phi\|_{H_{00}^{1 / 2}(\Gamma)} \cdot
$$

Proof. When the domain is decomposed into two subdomains, the estimate follows easily by a simple interpolation argument, using the well-known $H^{1}$ stability of the $L^{2}$ projection, cf. [19]. But the proof is not so trivial in general, since it is not clear how the interpolation argument can be applied to multi-subdomains with a cross-point case. The proof given below can also be found in [5].

To proceed, we first recall the following well-known estimate:

$$
\|u\|_{L^{2}\left(\partial \Omega_{i}\right)} \lesssim \epsilon^{-1}\|u\|_{L^{2}\left(\Omega_{i}\right)}+\epsilon\|u\|_{H^{1}\left(\Omega_{i}\right)}
$$

for any $\epsilon \in(0,1)$. A proof of this estimate can be found, for example, in [8].

We shall use the operator $R_{h}$, the $L^{2}$ projection from $L^{2}(\Omega)$ to $V_{0}^{h}(\Omega)$, and its approximation and stability property

$$
\left\|u-R_{h} u\right\|_{0, \Omega}+h\left\|R_{h} u\right\|_{1, \Omega} \lesssim h\|u\|_{1, \Omega} \quad \forall u \in H_{0}^{1}(\Omega) .
$$

It follows that

$$
\begin{aligned}
\| Q_{h} \phi & \left\|_{H_{00}^{1 / 2}(\Gamma)} \leq\right\| Q_{h} \phi-R_{h} \phi^{H}\left\|_{H_{00}^{1 / 2}(\Gamma)}+\right\| R_{h} \phi^{H} \|_{H_{00}^{1 / 2}(\Gamma)} \quad \text { (triangle inequality) } \\
& \lesssim h^{-1 / 2}\left\|Q_{h} \phi-R_{h} \phi^{H}\right\|_{0, \Gamma}+\left\|R_{h} \phi^{H}\right\|_{1, \Omega} \quad \text { (inverse inequality) } \\
& \lesssim h^{-1 / 2}\left\|\phi-R_{h} \phi^{H}\right\|_{0, \Gamma}+\left\|R_{h} \phi^{H}\right\|_{1, \Omega} \\
& \lesssim h^{-1}\left\|\phi^{H}-R_{h} \phi^{H}\right\|_{0, \Omega}+\left\|\phi^{H}-R_{h} \phi^{H}\right\|_{1, \Omega}+\left\|R_{h} \phi^{H}\right\|_{1, \Omega} \quad \text { (by (12)) } \\
& \lesssim\left\|\phi^{H}\right\|_{1, \Omega} \quad(\text { by }(13)) \\
& =\|\phi\|_{H_{00}^{1 / 2}(\Gamma) \quad \text { (by definition). } \quad \square}^{\square}
\end{aligned}
$$

Theorem 3.2. For all $u_{\Gamma}^{h} \in V_{\Gamma}^{h}$, we have

$$
\left(S_{h}^{-1} u_{\Gamma}^{h}, u_{\Gamma}^{h}\right) \equiv\left(T_{h} u_{\Gamma}^{h}, u_{\Gamma}^{h}\right) .
$$

Proof. From the definition of $T_{h}$, we see that

$$
\begin{aligned}
\left(T_{h} u_{\Gamma}^{h}, u_{\Gamma}^{h}\right) & =\left(S^{-1} u_{\Gamma}^{h}, u_{\Gamma}^{h}\right) \\
& =\left(S^{-1} u_{\Gamma}^{h}, S S^{-1} u_{\Gamma}^{h}\right) \\
& =\left\|S^{-1} u_{\Gamma}^{h}\right\|_{H_{00}^{1 / 2}(\Gamma)}^{2} \quad(\text { by }(7)) \\
& =\left\|u_{\Gamma}^{h}\right\|_{-1 / 2, \Gamma}^{2} \quad(\text { by }(7)) .
\end{aligned}
$$


From the definition of $S_{h}$, we see that

$$
\left(S_{h} u_{\Gamma}^{h}, u_{\Gamma}^{h}\right)=a\left(u_{h}^{H}, u_{h}^{H}\right) \equiv a\left(u^{H}, u^{H}\right)=\left\|u_{\Gamma}^{h}\right\|_{H_{00}^{1 / 2}(\Gamma)} ;
$$

here, $u^{H}$ is the harmonic extension of $u_{\Gamma}^{h}$.

Note that $S_{h}$ is a symmetric positive definite operator; we have

$$
\begin{aligned}
\left\|S_{h}^{-1 / 2} u_{\Gamma}^{h}\right\|^{2} & =\left(S_{h}^{-1} u_{\Gamma}^{h}, u_{\Gamma}^{h}\right) \\
& \leq\left\|S_{h}^{-1} u_{\Gamma}^{h}\right\|_{H_{00}^{1 / 2}(\Gamma)}\left\|u_{\Gamma}^{h}\right\|_{-1 / 2, \Gamma} \\
& \lesssim\left\|S_{h}^{1 / 2} S_{h}^{-1} u_{\Gamma}^{h}\right\|\left\|u_{\Gamma}^{h}\right\|_{-1 / 2, \Gamma} \quad(\text { by }(16)) \\
& =\left\|S_{h}^{-1 / 2} u_{\Gamma}^{h}\right\|\left\|u_{\Gamma}^{h}\right\|_{-1 / 2, \Gamma} .
\end{aligned}
$$

Hence, we get

$$
\left(S_{h}^{-1} u_{\Gamma}^{h}, u_{\Gamma}^{h}\right) \lesssim\left\|u_{\Gamma}^{h}\right\|_{-1 / 2, \Gamma}^{2} .
$$

On the other hand, for any $\phi \in H_{00}^{1 / 2}(\Gamma)$,

$$
\begin{aligned}
\left(u_{\Gamma}^{h}, \phi\right) & =\left(u_{\Gamma}^{h}, Q_{h} \phi\right)=\left\|S_{h}^{-1 / 2} u_{\Gamma}^{h}\right\|\left\|S_{h}^{1 / 2} Q_{h} \phi\right\| \\
& \lesssim\left\|S_{h}^{-1 / 2} u_{\Gamma}^{h}\right\|\left\|Q_{h} \phi\right\|_{H_{00}^{1 / 2}(\Gamma)} \quad(\text { by }(16)) \\
& \left.\lesssim\left\|S_{h}^{-1 / 2} u_{\Gamma}^{h}\right\|\|\phi\|_{H_{00}^{1 / 2}(\Gamma)} \quad \text { (by Lemma } 3.1\right) .
\end{aligned}
$$

Therefore, by the definition of $H^{-1 / 2}(\Gamma)$,

$$
\left\|u_{\Gamma}^{h}\right\|_{-1 / 2, \Gamma}^{2} \lesssim\left(S_{h}^{-1} u_{\Gamma}^{h}, u_{\Gamma}^{h}\right) .
$$

We now have that

$$
\left(S_{h}^{-1} u_{\Gamma}^{h}, u_{\Gamma}^{h}\right) \equiv\left\|u_{\Gamma}^{h}\right\|_{-1 / 2, \Gamma}^{2} .
$$

From (15) and (17), we obtain

$$
\left(S_{h}^{-1} u_{\Gamma}^{h}, u_{\Gamma}^{h}\right) \equiv\left(T_{h} u_{\Gamma}^{h}, u_{\Gamma}^{h}\right)
$$

3.2. Matrix representation. In this section, we shall demonstrate that the discrete Poincaré-Steklov operator $S_{h}$ defined in the previous subsection is closely related to the so-called Schur complement, and the discrete inverse operator $T_{h}$ can be written as a matrix whose entries are determined by the Green's function. As a result, we obtain a matrix that is spectrally equivalent to the inverse of the Schur complement.

Let the set of nodal points $\Omega^{h}=\left\{z_{i}\right\}$ be ordered in such a way that $\Gamma^{h}=\left\{z_{i} ; i=\right.$ $1,2, \ldots, N\}$. It is well known that the finite element discretization of the boundary value problem (1) can be reduced to the following linear algebraic system:

$$
\mathcal{A} \mu=\beta,
$$

where $\mathcal{A}=\left(a\left(\phi_{i}, \phi_{j}\right)\right)_{z_{i}, z_{j} \in \Omega^{h}}$ is the stiffness matrix and $\beta=\left(f, \phi_{i}\right)_{z_{i} \in \Omega^{h}}$. The vector $\mu$ corresponds to the nodal values of the finite element approximation.

In relation to $\Gamma^{h}$ and $\Omega^{h} \backslash \Gamma^{h}$, the stiffness matrix $\mathcal{A}$ can be written in a block form,

$$
\mathcal{A}=\left(\begin{array}{ll}
\mathcal{A}_{00} & \mathcal{A}_{01} \\
\mathcal{A}_{10} & \mathcal{A}_{11}
\end{array}\right)
$$


and the Schur complement is

$$
\mathcal{S}=\mathcal{A}_{00}-\mathcal{A}_{01} \mathcal{A}_{11}^{-1} \mathcal{A}_{10}
$$

A direct computation leads to another expression for the elements of $\mathcal{S}=$ $\left(s_{i j}\right)_{N \times N}$ :

$$
s_{i j}=\left(\left.S_{h} \phi_{i}\right|_{\Gamma},\left.\phi_{j}\right|_{\Gamma}\right), \quad i, j \in\{1,2, \ldots, N\} .
$$

Let $Q_{h}$ or $Q_{h}^{\prime}$ denote the $L^{2}$ projection from $L^{2}(\Gamma)$ to $V_{\Gamma}^{h}$, and $G^{h}\left(z, z^{\prime}\right)$ denote a function in the tensor product space of $V_{\Gamma}^{h}$ and $V_{\Gamma}^{h}$ as follows:

$$
G^{h}\left(z, z^{\prime}\right)=\left(Q_{h} Q_{h}^{\prime} G^{0}\right)\left(z, z^{\prime}\right) .
$$

Here $Q_{h}$ and $Q_{h}^{\prime}$ mean that the action is with respect to $z$ and $z^{\prime}$, respectively.

Theorem 3.3. Define $\mathcal{T}=\left(t_{i j}\right) \in \mathbf{R}^{N \times N}$ by

$$
t_{i j}=G^{h}\left(z_{i}, z_{j}^{\prime}\right), \quad i, j \in\{1,2, \ldots, N\} .
$$

Then $\mathcal{T}$ is a symmetric positive definite matrix such that

$$
\kappa(\mathcal{T S}) \equiv 1 \text {. }
$$

Here $\kappa$ is the condition number of the relevant matrix.

Proof. Let $\mathcal{M}=\left(\left(\phi_{i}, \phi_{j}\right)_{\Gamma}\right)_{z_{i}, z_{j} \in \Gamma^{h}}$ be the mass matrix on the interface, and $\mu_{\Gamma}$ be the vector corresponding to the restriction of the finite element function $u_{\Gamma}^{h}$ to $\Gamma^{h}$; it can be verified that

$$
\left(S_{h}^{-1} u_{\Gamma}^{h}, u_{\Gamma}^{h}\right)=\left(\mathcal{M S}^{-1} \mathcal{M} \mu_{\Gamma}, \mu_{\Gamma}\right)
$$

and

$$
\left(T_{h} u_{\Gamma}^{h}, u_{\Gamma}^{h}\right)=\left(\mathcal{M T} \mathcal{M} \mu_{\Gamma}, \mu_{\Gamma}\right) .
$$

By Theorem 3.2, the conclusion of the theorem follows immediately.

In practical computation, we propose to use the following simpler formula to compute the elements of the preconditioner $\hat{\mathcal{T}}=\left(\hat{t}_{i j}\right) \in \mathbf{R}^{N \times N}$, with

$$
\hat{t}_{i j}=G^{0}\left(z_{i}, z_{j}\right) \quad(i \neq j), \quad \hat{t}_{i i}=\frac{1}{\delta} \int_{z^{\prime} \in \Gamma, \operatorname{dist}\left(z^{\prime}, z_{i}\right) \leq h / 2} G^{0}\left(z_{i}, z^{\prime}\right) d z^{\prime} .
$$

Here, $\delta$ is the measure of the set $\left\{z^{\prime} \in \Gamma: \operatorname{dist}\left(z^{\prime}, z_{i}\right) \leq h / 2\right\}$.

The modified preconditioner (20) is expected to be a good approximation to $\mathcal{T}$ given by (19) because of the approximation property and "local" property of finite element $L^{2}$ projections. Unfortunately, we have not been able to theoretically justify such a modification. Nevertheless, we shall provide several numerical experiments in the following section to demonstrate its efficiency.

Remark 2. When the preconditioned conjugate gradient method is used, the action of the preconditioner needs to be computed. As the preconditioning matrix is full, a direct matrix vector multiplication requires $O\left(h^{-2}\right)$ operations. Although this complexity is optimal when this preconditioner is used in a domain decomposition method for two-dimensional computation, with more efficient techniques (such as multi-pole methods, see [9]), it is possible to evaluate the action of this matrix with $O\left(h^{-1}|\log h|\right)$ operations. 
Remark 3. In the above discussion, the domain $\Omega$ was supposed to be the unit disk, and the matrix $\mathcal{T}$ determined by the Green's function of the unit disk was proved to be spectrally equivalent to the inverse of the Schur complement reduced from the stiffness matrix on the unit disk. If the domain $\Omega$ is not the unit disk, but the interface $\Gamma$ of the unit disk can still decompose $\Omega$ into subdomains, it can be proved that the Schur complement reduced from the unit disk is spectrally equivalent to the Schur complement reduced from $\Omega$. Therefore, $\mathcal{T}$ is still spectrally equivalent to the inverse of the Schur complement of $\Omega$.

Furthermore, when $\Gamma$ is composed of some segments which meet at the crosspoint, and the length of these segments are roughly the same, and the numbers of nodal points in different segments are of the same order of magnitude, it can still be proved that $\mathcal{T}$ is spectrally equivalent to the inverse of the Schur complement.

\section{Applications And numericAl EXAmples}

The preconditioner we proposed in this paper can be combined with other wellknown domain decomposition methods. In this section, we shall give some examples for such applications. But, first, we shall report some numerical examples for our new preconditioner itself. We shall consider the model boundary value problem (1) discretized by triangular linear finite elements defined on a uniform triangulation on $\Omega$.

4.1. Basic numerical tests. To test our new preconditioner, we first take the Poisson equation on a rectangular domain $(-1,1) \times(-2,2)$ which is decomposed into two subdomains interfaced at the interval $(-1,1)$ on the $x$-axis. The result for preconditioner $\mathcal{T}$ defined by (19) is given in Table 1 and the result for preconditioner $\hat{\mathcal{T}}$ is given in Table 2. In these tables, $N$ is the number of nodal points on the interface.

TABLe 1. Preconditioner (19) for the two-subdomain case

\begin{tabular}{lllllll}
\hline \hline$N$ & 3 & 7 & 15 & 31 & 63 & 127 \\
$\kappa(\mathcal{T S})$ & 2.322 & 2.886 & 3.042 & 3.083 & 3.094 & 3.097 \\
\hline
\end{tabular}

TABLE 2. Preconditioner (20) for the two-subdomain case

\begin{tabular}{llllllllll}
\hline \hline$N$ & 3 & 7 & 15 & 31 & 63 & 127 & 255 & 511 & 1023 \\
$\kappa(\hat{\mathcal{T}} \mathcal{S})$ & 1.265 & 1.369 & 1.399 & 1.407 & 1.412 & 1.414 & 1.414 & 1.414 & 1.414 \\
\hline
\end{tabular}

As we see from Tables $1-2$, the condition number $\kappa(\mathcal{T} \mathcal{S})$ is bounded independently of $h$, as predicted by our theory. And $\kappa(\hat{\mathcal{T}} \mathcal{S})$ is actually smaller than $\kappa(\mathcal{T} \mathcal{S})$.

To test our preconditioner for the cross-point case, we take a unit square and divide it into four subsquares with a cross-point at the center. Table 3 , where $N$ is the number of nodal points in the interior of each of the four segments of the interface, contains the results for the preconditioner $\hat{\mathcal{T}}$ defined by the simplified formula $(20)$. As can be seen, $\kappa(\hat{\mathcal{T}} \mathcal{S})$ is very small indeed. 
TABle 3. Preconditioner (20) for the cross-point case

\begin{tabular}{llllllllll}
\hline \hline$N$ & 3 & 5 & 10 & 15 & 20 & 40 & 60 & 80 & 100 \\
$\kappa(\hat{\mathcal{T}} \mathcal{S})$ & 1.263 & 1.280 & 1.281 & 1.284 & 1.284 & 1.282 & 1.281 & 1.280 & 1.280 \\
\hline
\end{tabular}

To demonstrate the robustness of our preconditioner for the cross-point case, we test a cross-point example with different numbers of nodal points in the four interface segments around it. Table 4 , where $N_{1}, N_{2}, N_{3}$ and $N_{4}$ are numbers of nodal points in the west, east, north and south direction, respectively, shows that our new preconditioner is also optimal even if the grid is not uniform.

TABLe 4. Preconditioner (20) with nonuniform mesh around the cross-point

\begin{tabular}{llllll}
\hline \hline$N_{1}$ & 5 & 11 & 23 & 47 & 95 \\
$N_{2}$ & 10 & 21 & 43 & 87 & 175 \\
$N_{3}$ & 7 & 15 & 31 & 63 & 127 \\
$N_{4}$ & 15 & 31 & 63 & 127 & 255 \\
$\kappa(\hat{\mathcal{T}} \mathcal{S})$ & 1.80 & 1.86 & 1.89 & 1.92 & 1.94 \\
\hline
\end{tabular}

4.2. Multi-subdomain decomposition without overlappings. We shall now report numerical examples for applying our new preconditioner together with two well-known nonoverlapping domain decomposition methods: the substructuring method and the vertex space method.

Let $\Omega$ be a polygonal domain together with a coarse-grid triangulation $T^{0}$ with mesh size $h_{0}$. A finite element triangulation $T^{h}$ with mesh size $h$ is obtained by refining $T^{0}$. The coarse mesh $T_{0}$ is viewed as a domain decomposition of $\Omega$, and the mesh line of $T^{0}$ is then the interface $\Gamma$ of the decomposition. Let $\Omega^{0}$ and $\Omega^{h}$ denote the set of nodal points of the triangulations $T^{0}$ and $T^{h}$, and $S^{0}$ and $V_{0}^{h}$ the finite element function spaces corresponding to $T^{0}$ and $T^{h},\left\{\phi_{i}^{0}, z_{i} \in \Omega^{0}\right\}$ and $\left\{\phi_{i}, z_{i} \in \Omega^{h}\right\}$ the set of finite element basis functions of $S^{0}$ and $V^{h}$, respectively, and $\mathcal{A}^{0}$ the stiffness matrix corresponding to $T^{0}$.

The set of nodal points on the interface $\Gamma^{h}=\Gamma \cap \Omega^{h}$ can be divided into several mutually disjoint subsets,

$$
\Gamma^{h}=\Gamma^{0} \cup\left(\bigcup_{l=1}^{m} \Gamma_{l}^{h}\right),
$$

where $\Gamma^{0}$ is the set of coarse-grid nodal points (also cross-points), $\Gamma_{l}^{h} \subset \Gamma^{h}$ (l= $1, \ldots, m)$ consists of, say, $k_{1}$ nodal points in the interior of a segment of the interface.

Substructuring method. The first multi-subdomain preconditioner considered is an additive variant of the substructuring preconditioner of Bramble, Pasciak and Schatz [2]:

$$
\mathcal{T}=\mathcal{I}_{0} \mathcal{A}_{0}^{-1} \mathcal{I}_{0}^{t}+\sum_{e \in \mathcal{E}} \mathcal{I}_{e} \mathcal{T}_{e} \mathcal{I}_{e}^{t}
$$


Here, $\mathcal{I}_{0}=\left(\alpha_{i j}\right)$ is the prolongation such that $\alpha_{i j}=\phi_{j}^{0}\left(z_{i}\right), \mathcal{I}_{e}$ is the zero extension operator from $\Gamma_{l}^{h}$ to $\Gamma^{h}$, and $\mathcal{T}_{e}$ are the matrices defined by $(20)$, and $\mathcal{E}$ is the set of all coarse-grid element edges (namely the edges from domain decomposition interface).

Table 5 contains the results for the condition numbers of $\hat{\mathcal{T}} \mathcal{S}$, which grow at the rate of the polylogarithm of the number $k_{1}$ of nodal points in the segments of interface.

TABLE 5. A variant of the substructuring preconditioner (21)

\begin{tabular}{llllllll}
\hline \hline$p$ & 4 & 4 & 4 & 4 & 4 & 4 & 4 \\
$k_{1}$ & 3 & 5 & 10 & 20 & 40 & 80 & 100 \\
$\kappa$ & 4.54 & 6.00 & 8.61 & 12.0 & 16.0 & 20.8 & 22.5 \\
\hline
\end{tabular}

Note that the growth of condition numbers shown in Table 5 is due to the preconditioner (21) itself and it agrees with the theoretical prediction in [2]. In fact the numerical values shown in Table 5 are also very close to those reported in [2]. In this particular application with the uniform grid, however, our Green's function approach may not be, computationally, as efficient as the FFT approach used in [2], but such a simple situation is not the intended application of our method; nevertheless, it indeed shows the efficiency of our method in this case. More interesting applications of the method to the cross-point case are given below.

Vertex space method. The vertex space method is a modification of the above substructuring method, where an additional space is introduced, called vertex space, around each cross-point in the domain decomposition (cf. Smith [14], and also Matsokin and Nepomnyaschikh [11]). More specifically, at each cross-point in $\Gamma^{0}$, we select a subset of $\Gamma^{h}$ around it, which contains about $k_{2}$ points along each segment connecting to this cross-point; this set is denoted by $\Gamma_{v}^{h}(v \in \mathcal{V})$. The vertex space preconditioner can be written as

$$
\mathcal{T}=\mathcal{I}_{0} \mathcal{A}_{0}^{-1} \mathcal{I}_{0}^{t}+\sum_{v \in \mathcal{V}} \mathcal{I}_{v} \mathcal{I}_{v} \mathcal{I}_{v}^{t}+\sum_{e \in \mathcal{E}} \mathcal{I}_{e} \mathcal{T}_{e} \mathcal{I}_{e}^{t} .
$$

Compared with [2], the above preconditioner has additional terms involving $v \in$ $\mathcal{V}$. Here, $\mathcal{I}_{v}$ is the zero extension operator from $\Gamma_{v}^{h}$ to $\Gamma^{h}$, and $\mathcal{T}_{v}$ are the matrices defined by (20) for the cross-point case.

According to the theory of Dryja and Widlund [7], the preconditioner (22) admits the following estimate:

$$
\kappa(\mathcal{T S}) \lesssim \log ^{2}\left(1+k_{1} / k_{2}\right) .
$$

Note that the preconditioner (22) is optimal if the number of nodal points in $\Gamma_{v}^{h}$ is proportional to the number of nodal points in $\Gamma_{l}^{h}$.

In our test, we decompose the unit square into $p \times p$ subsquares, and each subsquare contains $k_{1} \times k_{1}$ nodal points in its interior. On the $(p-1) \times(p-1)$ cross-points, we select $k_{2}$ points in each of the four segments around it to form the cross-point components in the preconditioner.

Tables 6-9 contain results with a different number of subdomains, different number of nodal points in subdomains and different number of nodal points around a 
cross-point, respectively. From these data, we can tell the role of different parts of the preconditioner, and the results appear to be in agreement with the estimate $(23)$.

TABLE 6 . The effect of $k_{2}$ for preconditioner (22)

\begin{tabular}{llllllll}
\hline \hline$p$ & 4 & 4 & 4 & 4 & 4 & 4 & 4 \\
$k_{1}$ & 40 & 40 & 40 & 40 & 40 & 40 & 40 \\
$k_{2}$ & 1 & 2 & 4 & 8 & 12 & 16 & 20 \\
$\kappa$ & 4.96 & 4.32 & 3.83 & 3.01 & 3.03 & 3.02 & 2.96 \\
\hline
\end{tabular}

Table 6 contains results for a fixed number of subdomains and a fixed number of nodal points in subdomains, but variable number of nodal points around cross-points. The results show that the size of the cross-point component in the preconditioner affects the condition number very weakly (see (23)).

TABLE 7. Results for very small vertex spaces

\begin{tabular}{lllllll}
\hline \hline$p$ & 10 & 10 & 10 & 10 & 10 & 10 \\
$k_{1}$ & 10 & 20 & 40 & 60 & 80 & 100 \\
$k_{2}$ & 3 & 3 & 3 & 3 & 3 & 3 \\
$\kappa$ & 2.93 & 2.99 & 3.91 & 4.55 & 5.00 & 5.40 \\
\hline
\end{tabular}

Table 7 shows that when the scale of the cross-point component in the preconditioner is kept fixed, the condition number varies slowly with respect to the scale of subproblems also.

TABLE 8. Optimality of the preconditioner (22)

\begin{tabular}{lllllllllllll}
\hline \hline$p$ & 5 & 10 & 20 & 30 & 40 & 50 & 60 & 70 & 80 & 90 & 100 & 100 \\
$k_{1}$ & 5 & 5 & 5 & 5 & 5 & 5 & 5 & 5 & 5 & 5 & 5 & 10 \\
$k_{2}$ & 1 & 1 & 1 & 1 & 1 & 1 & 1 & 1 & 1 & 1 & 1 & 3 \\
$\kappa$ & 2.78 & 2.83 & 2.85 & 2.85 & 2.85 & 2.85 & 2.85 & 2.85 & 2.86 & 2.86 & 2.31 & 2.42 \\
\hline
\end{tabular}

Table 8 shows that when there is the coarse-grid component in the preconditioner, the preconditioner will be optimal and the condition number is independent of the size of the coarse grid only if the local parameter $\frac{k_{2}}{k_{1}}$ is kept fixed.

TABLE 9. Result without a coarse-grid space

\begin{tabular}{llllllll}
\hline \hline$p$ & 5 & 10 & 20 & 30 & 40 & 50 & 60 \\
$k_{1}$ & 5 & 5 & 5 & 5 & 5 & 5 & 5 \\
$k_{2}$ & 1 & 1 & 1 & 1 & 1 & 1 & 1 \\
$\kappa$ & 35.2 & 137 & 545 & 1224.1 & 2175.3 & 3398.4 & 4892.0 \\
\hline
\end{tabular}


Table 9 contains results for a preconditioner not using the coarse-grid space, and it shows the importance of the coarse-grid component in the preconditioning.

\section{ACKNOWLEDGEMENT}

The authors wish to thank Dr. Dehao Yu for discussions.

\section{REFERENCES}

1. J. H. Bramble, J. E. Pasciak, and A. H. Schatz. The construction of preconditioners for elliptic problems by substructuring, I. Math. Comp., 47:103-134, 1986. MR 87m:65174

2. . An iterative method for elliptic problems on regions partitioned into substructures. Math. Comp., 46:361-369, 1986. MR 88a:65123

3. Comp., 49:1-16, 1987. MR 88j:65248

4. Comp., 53:1-24, 1989. MR 89m:65098

5. J. H. Bramble, J. E. Pasciak, and J. Xu. A multilevel preconditioner for domain decomposition boundary systems. In Proceeding of 10th International Conference on Computing Methods in Applied Sciences and Engineering. INRIA, 1991.

6. M. Dryja. A method of domain decomposition for 3-D finite element elliptic problems. In R. Glowinski, G. H. Golub, G. A. Meurant, and J. Périaux, editors, First International Symposium on Domain Decomposition Methods for Partial Differential Equations, Philadelphia, PA, 1988. SIAM. MR 90b:65200

7. M. Dryja and O. B. Widlund. Domain decomposition algorithms with small overlap. SIAM J. Sci. Statist. Comput., 15:604-620, 1994. MR 95d:65102

8. P. Grisvard. Elliptic problems in nonsmooth domains. Pitman Advanced Publishing Program, Boston, 1985. MR 86m:35044

9. L. Greengard J. Carrier and V. Rokhlin. A fast adaptive multipole algorithm for particle simulations. SIAM J. Sci. Statist. Comput., 9:669-686, 1988. MR 89e:65008

10. J. L. Lions and E. Magenes. Nonhomogeneous Boundary Value Problems and Applications, volume I. Springer, New York, Heidelberg, Berlin, 1972. MR 50:2670

11. A. M. Matsokin and S. V. Nepomnyashchikh. A Schwarz alternating method in a subspace. Soviet Mathematics, 29:78-84, 1985

12. Norms in the space of traces of mesh functions. Soviet J. Numer. Anal. Math. Modelling, 3:199-216, 1988.

13. J. Nečas. Les Méthodes directes en Théorie des Équations Elliptiques. Academia, Prague, 1967. MR 37:3168

14. B. F. Smith. An optimal domain decomposition preconditioner for the finite element solution of linear elasticity problems. SIAM J. Sci. Statist. Comput., 13:364-378, 1992. MR 92g:73080

15. B. F. Smith and O. B. Widlund. A domain decomposition algorithm using a hierarchical basis. SIAM J. Sci. Statist. Comput., 11:1212-1220, 1990. MR 91m:65125

16. C. H. Tong, T. F. Chan and C. J. Kuo. A domain decomposition preconditioner based on a change to a multilevel nodal basis. SIAM J. Sci. Statist. Comput., 12:1486-1495, 1991. MR 92i: 65070

17. J. Xu. Theory of multilevel methods. Ph.D. thesis, Cornell University, May 1989.

18. I Iterative methods by space decomposition and subspace correction. SIAM Review, 34:581-613, 1992. MR 93k:65029

19. _ Multigrid and domain decomposition methods. Research Monograph (preprint), 1995.

Department of Mathematics, Penn State University. University Park, Pennsylvania 16802

E-mail address: xu@math.psu.edu

State Key Laboratory of Scientific and Engineering Computing, Computing Center, Chinese Academy of Sciences, Beijing 100080, P.R. China

E-mail address: zhang_s@math.psu.edu 